\title{
Mosaic trisomy 16 in a live newborn infant
}

\author{
N J Gilbertson, J W Taylor, I Z Kovar
}

\begin{abstract}
Trisomy 16 is thought to be incompatible with fetal survival. A boy with mosaic trisomy 16 who lived for 11 weeks is reported. Chromosome analysis was carried out on skin fibroblasts grown during life and confirmed on samples taken at necropsy. We believe that this is the first report of mosaic trisomy 16 that has been confirmed by cytogenetic banding.
\end{abstract}

Trisomy 16 is the most common autosomal anomaly seen in early aborted fetuses, but has been thought to be incompatible with full embryonic development; empty sacs and disorganised embryos are usually seen. ${ }^{1}$ There are reports of putative full and mosaic trisomy 16 but none confirmed by cytogenetic banding studies. We report an abnormal baby boy born alive with mosaic trisomy 16 who survived for 11 weeks.

\section{Case report}

A baby boy was born at 39 weeks' gestation to healthy Chinese parents. During the pregnancy symmetrical intrauterine growth retardation and polyhydramnios were noted; delivery was by caesarean section because of fetal distress and the growth retardation.

The baby was initially cyanosed with regular shallow respiratory efforts and a heart rate of 60 beats/minute; he responded well to oxygen given by face mask. Craniofacial and musculoskeletal dysmorphic features were immediately apparent as was clinical growth retardation; he weighed $1590 \mathrm{~g}$ at birth, was $40 \mathrm{~cm}$ long, and had a head circumference of $31.5 \mathrm{~cm}$ (all below the third centile). The abnormalities noted are listed in the table; the infant is shown in the figure.

The baby required ventilatory support for the first three weeks because of respiratory distress, and was then maintained with oxygen given by headbox for a further three weeks. The respiratory insufficiency was thought to be caused by restriction from kyphoscoliosis. A ventilationperfusion lung scan carried out at 8 weeks showed equal ventilation bilaterally, but with reduced ventilation in the upper zones. There was a discrepancy between ventilation and perfusion in the right upper zone, which was underperfused.

A loud pulmonary second sound and forceful left parasternal impulse were noted at birth; a pansystolic murmur was audible at the lower left sternal edge from 4 weeks. An echocardiogram showed a small perimembranous ventricular septal defect, which seemed to be closing by apposition of the tricuspid valve tissue.
Phenotypic abnormalities
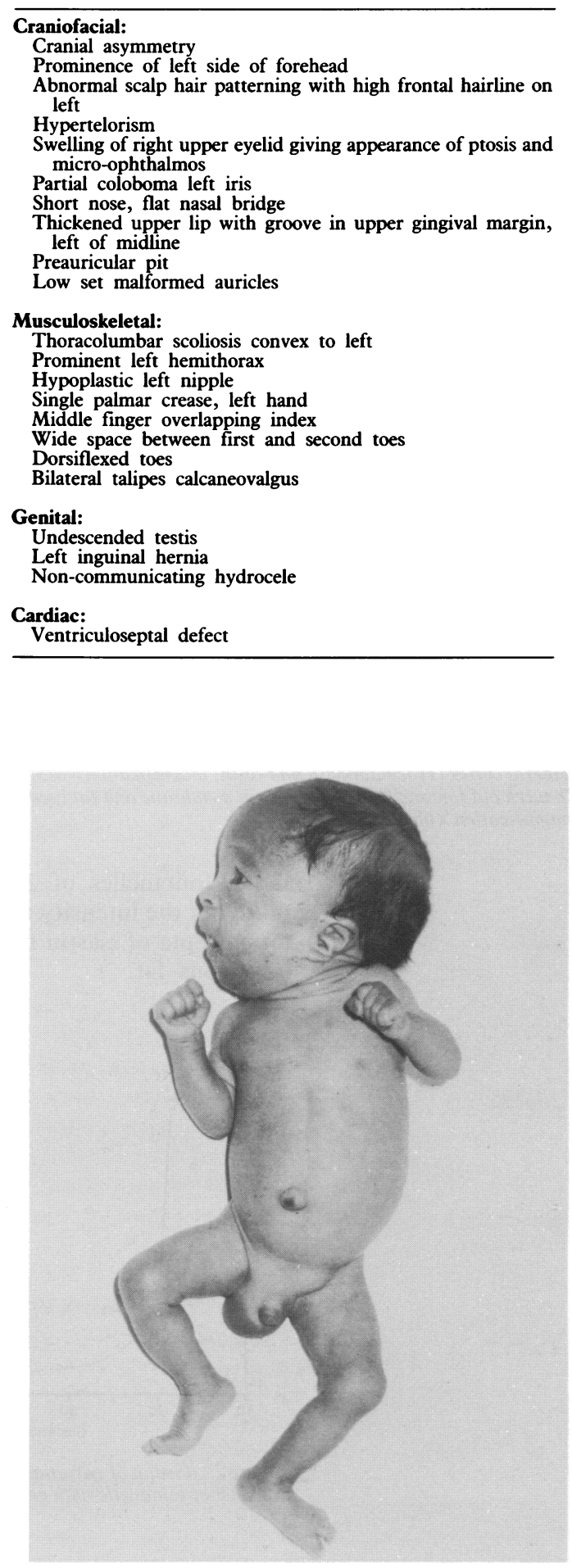

Appearance of infant with mosaic trisomy 16

The infant was fed initially by nasogastric tube, and subsequently by bottle from the age of 8 weeks. Despite a maximal milk intake his weight gain was slow and erratic. He gained just 
over a kilogram during the 11 weeks of his life, and weighed $2610 \mathrm{~g}$ at the time of his death.

He was always socially unresponsive and was never seen to smile. He was hypotonic with no head control ('rag doll-like'). He was visually attentive and had normal non-corneal electroretinograms, with bilateral cortical visual evoked potentials to flash stimuli. He did not respond to sound; auditory evoked potentials at eight weeks were inconsistent. An electroencephalogram showed no abnormality.

At 11 weeks his inguinal hernia became difficult to reduce and surgical repair was indicated. He was by that time clinically stable, and had been breathing air spontaneously for five weeks. The endotracheal intubation for general anaesthesia was extremely difficult, and the patient became hypoxic and had a cardiorespiratory arrest. Resuscitation was unsuccessful.

Necropsy was performed at the request of the coroner and left sided hypertrophy of the cornua of the hyoid bone and thyroid cartilage was found, together with pronounced hypertrophy of the left arytenoid cartilage which seemed to have caused partial obstruction of the glottis. There was a deep cleft between the hypertrophic left arytenoid area and the epiglottis. The vocal cords were asymmetrical, particularly on the left, and there was stenosis in the midsection of the trachea. A small aberrant left bronchus that was barely patent was present above the left main bronchus, and the right bronchus was elongated. There were no other findings of note except for the small perimembranous ventriculoseptal defect previously noted, and the dysmorphic features.

\section{Cytogenetic studies}

Chromosome analyses using G banding were carried out on 72 hour lymphocyte cultures. A total of 20 metaphases were analysed and no abnormality was found, suggesting a karyotype to be $46, \mathrm{XY}$.

The chromosomal analysis was repeated using fibroblast cultures from a skin biopsy specimen taken from the left thigh during the second week of life. $G$ banding of the chromosome preparations indicated two different cell lines. Of a total 21 metaphases examined, six showed a normal male karyotype, $46, \mathrm{XY}$. The remaining 15 metaphases were trisomic for the whole of chromosome number 16, with a chromosome count of 47 . The proband karyotype was $46, \mathrm{XY} / 47, \mathrm{XY}+16$.

The cell line which was trisomic for chromosome 16 was less stable in culture than the normal cell line. The proportion of trisomic cells to normal cells decreased with increasing time in culture, especially after subculturing the fibroblasts. The trisomy 16 mosaicism was confirmed in fibroblasts taken from skin and muscle biopsy specimens taken at necropsy. Roughly half the fibroblasts were trisomic for chromosome 16, and the rest showed a normal karyotype. Further tissues were not cultured for chromosomal studies and so the differential distribution of trisomic cells could not be examined. Studies of both parents showed normal karyotypes.

\section{Discussion}

We believe that this is the first report of a mosaic trisomy 16 confirmed by cytogenetic banding, and we have described phenotype abnormalities in addition to those noted previously in potentially similar cases.

Trisomy 16 has been reported as the most common autosomal trisomy in aborted fetuses. There have been isolated reports of $16-$ mosaicism in adults; a physically and mentally normal Japanese father of an aborted fetus that was trisomic for chromosome 16 had $3 \%$ of his white cells and $15 \%$ of his skin fibroblasts trisomic for chromosome $16 .^{2}$ Some of the abnormalities that were seen in our proband-including kyphoscoliosis, breast hypoplasia, and asymmetric musculoskeletal developmenthave been described previously, ${ }^{3}$ but in these reports of mosaic trisomy 16 and in two of full trisomy 16 chromosome analysis was carried out without modern banding techniques. ${ }^{45}$

There have been reports of infants surviving with trisomy of either $16 \mathrm{p}$ or $16 \mathrm{q}$ material confirmed by GTG banding; ${ }^{6}$; partial trisomy 16 may also occur with familial 16:21 translocation. Patients with partial trisomy 16 all have multiple congenital anomalies and with one exception have died within the first year of life. As both trisomy $16 p$ and $16 q$ are compatible with intrauterine life and a limited degree of postnatal survival Roberts and Duckett concluded that it must be the combined effects of additional long and short arms which render the full trisomy invariably lethal. ${ }^{6}$ It is noteworthy that trisomy 16 has been detected in chorion villus biopsy specimens from a number of cases in which the fetus itself was unaffected, indicating that the mosaic is formed in the early embryo and that the trophoblast can function normally in the presence of this trisomy. Our proband has some features in common with those seen in partial trisomies, namely skull asymmetry, auricular abnormalities, single palmar crease, cryptorchidism, inguinal hernia, ventriculoseptal defect, failure to thrive, and developmental retardation. Other features of this infant that have not been noted previously include coloboma, abnormal patterning of scalp hair, and asymmetry of laryngeal structures.

This infant had a higher proportion of abnormal cells than suggested by the unconfirmed reports of mosaic trisomy 16 . It remains to be determined what proportion of abnormal cells can exist before a mosaic trisomy becomes lethal, or if a full trimsomy 16 is compatible with survival. Consideration of this baby's anomalies may assist in delineation of a phenotype for the trisomy of chromosome 16 material.

1 Creasy MR, Crolla JA, Alberman ED. Cytogenetic study of human spontaneous abortions using banding techniques. human spontaneous abortions

2 Arakaki DT, Waxman SH. Trisomy 16 in a mosaic carrie father and his aborted fetus. 7 Med Genet 1969;6:85-8 father and his aborted fetus. F Med Genet 1969;6:85-8.

16+. $\mathcal{F}$ Med Genet 1971;8:123-5.
Lewis FJW, Hyman JM, McTaggart M, Poulding RH. Lewis FJW, Hyman JM, McTaggart M, Pould
Trisomy of autosome 16. Nature 1963;199:404.

5 Melnyk J, Thompson H, Hecht F. Cytogenic studies in an apparent trisomy 16 (47XY 16+). Mammalian Chromosome Newsletter 1968;9:39-40.

6 Roberts SH, Duckett DP. Trisomy 16p in a liveborn infant and a review of partial and full trisomy 16. $\mathcal{J}$ Med Genet 1978;15:375-81. 